\title{
Chromosomal microarray testing in adults with intellectual disability presenting with comorbid psychiatric disorders
}

\author{
Kate Wolfe ${ }^{\star,}$, André Strydom ${ }^{1}$, Deborah Morrogh ${ }^{2}$, Jennifer Carter ${ }^{2}$, Peter Cutajar ${ }^{3}$, Mo Eyeoyibo ${ }^{4}$, \\ Angela Hassiotis ${ }^{1}$, Jane McCarthy ${ }^{5}$, Raja Mukherjee ${ }^{6}$, Dimitrios Paschos ${ }^{7}$, Nagarajan Perumal ${ }^{8}$, Stephen $\operatorname{Read}^{9}$, \\ Rohit Shankar ${ }^{10}$, Saif Sharif ${ }^{11}$, Suchithra Thirulokachandran ${ }^{12}$, Johan H Thygesen ${ }^{1}$, Christine Patch ${ }^{13}$, \\ Caroline Ogilvie $^{13}$, Frances Flinter ${ }^{13}$, Andrew McQuillin ${ }^{1}$ and Nick Bass ${ }^{1}$
}

\begin{abstract}
Chromosomal copy-number variations (CNVs) are a class of genetic variants highly implicated in the aetiology of neurodevelopmental disorders, including intellectual disabilities (ID), schizophrenia and autism spectrum disorders (ASD). Yet the majority of adults with idiopathic ID presenting to psychiatric services have not been tested for CNVs. We undertook genomewide chromosomal microarray analysis (CMA) of 202 adults with idiopathic ID recruited from community and in-patient ID psychiatry services across England. CNV pathogenicity was assessed using standard clinical diagnostic methods and participants underwent comprehensive medical and psychiatric phenotyping. We found an $11 \%$ yield of likely pathogenic CNVs (22/202). CNVs at recurrent loci, including the 15q11-q13 and 16p11.2-p13.11 regions were most frequently observed. We observed an increased frequency of $16 \mathrm{p} 11.2$ duplications compared with those reported in single-disorder cohorts. CNVs were also identified in genes known to effect neurodevelopment, namely NRXN1 and GRIN2B. Furthermore deletions at 2q13, 12q21.2-21.31 and 19q13.32, and duplications at 4p16.3, 13q32.3-33.3 and Xq24-25 were observed. Routine CMA in ID psychiatry could uncover $\sim 11 \%$ new genetic diagnoses with potential implications for patient management. We advocate greater consideration of CMA in the assessment of adults with idiopathic ID presenting to psychiatry services.
\end{abstract}

European Journal of Human Genetics (2017) 25, 66-72; doi:10.1038/ejhg.2016.107; published online 21 September 2016

\section{INTRODUCTION}

Intellectual disability (ID) is defined as significant impairments in intellectual and adaptive functioning with onset before the age of 18 years. ID is a clinically heterogeneous disorder with a range of genetic and environmental causes. Genetic causes include aneuploidies, copynumber variations (CNVs) and single-nucleotide variants in specific genes. There are a wide range of environmental causes, notably perinatal infection and hypoxic injury. For $\sim 50 \%$ of individuals with ID the cause is unknown. ${ }^{1}$ There is an increased prevalence of psychiatric disorders in adults with ID, for example the point prevalence of psychosis has been estimated as 10 times higher than the general population. ${ }^{2}$ In the United Kingdom adult ID psychiatry is a specialist field. Investigation of the cause of ID/developmental delay (DD) predominately occurs at onset in childhood and there is no formalised system of diagnostic review. Genetic testing in adulthood can be carried out by ID psychiatrists and other treating clinicians, such as neurologists. Clinical genetics services are organised regionally in the UK and treating clinicians can make onward referrals for patients and families. Screening for genetic causes of ID has advanced from G-banded karyotyping to high-resolution genome-wide chromosomal microarray analysis (CMA), which is often the recommended first-tier cytogenetic test for DD/ID. ${ }^{3}$

CMA encompasses array comparative genomic hybridisation $(\mathrm{aCGH})$ and single-nucleotide polymorphism arrays, which detect submicroscopic losses or gains of genetic material known as CNVs. The clinical relevance of $\mathrm{CNV}$ events is determined by factors such as the inheritance pattern, size of the CNV, number of genes deleted or duplicated, likely functional consequence of gene disruption and presence of the $\mathrm{CNV}$ in healthy control data sets. ${ }^{3}$ Recurrent CNVs are found in regions of the genome harbouring simple repetitive DNA sequences known as low copy repeats. Propensity to recombination errors in these regions give rise to some of the well-known syndromic forms of ID, for example the recurrent deletions at the 15q11-q13 region which cause Prader Willi or Angelman's syndrome. CNVs can also be classed as pathogenic when there is a disruption of genes involved in important neurodevelopmental functions, such as neurotransmission. ${ }^{4}$

A CNV 'morbidity map' has identified $70 \mathrm{CNV}$ regions significantly associated with DD/ID in 29085 children; however, comparable data on adults with ID are limited. ${ }^{4}$ Study of research cohorts with

${ }^{1}$ Division of Psychiatry, University College London, London, UK; ${ }^{2}$ North East Thames Regional Genetics Service Laboratory, London, UK; ${ }^{3}$ Nottinghamshire Healthcare NHS Foundation Trust, Nottingham, UK; ${ }^{4}$ Kent and Medway NHS and Social Care Partnership Trust, Kent, UK; ${ }^{5}$ East London NHS Foundation Trust, London, UK; ${ }^{6}$ Surrey and Borders Partnership NHS Foundation Trust, Surrey, UK; ${ }^{7}$ Camden and Islington NHS Foundation Trust, London, UK; ${ }^{8}$ Cumbria Partnership NHS Foundation Trust, Cumbria, UK; ${ }^{9}$ South West Yorkshire Partnership NHS Foundation Trust, Yorkshire, UK; ${ }^{10}$ Cornwall Partnership NHS Foundation Trust, Cornwall, UK; ${ }^{11}$ Southern Health NHS Foundation Trust, Southampton, UK; ${ }^{12}$ Coventry and Warwickshire Partnership NHS trust, Coventry, UK; ${ }^{13}$ Guy's and St Thomas' NHS Foundation Trust, London, UK

${ }^{*}$ Correspondence: Ms K Wolfe, Molecular Psychiatry Laboratory, Division of Psychiatry, University College London, Rockefeller Building, Gower Street, London, WC1E 6BT, UK. Tel: +44 207679 6562; Fax: +44 203108 2194; E-mail: k.wolfe@ucl.ac.uk

Received 8 February 2016; revised 23 June 2016; accepted 28 June 2016; published online 21 September 2016 
schizophrenia and ASD have also revealed rare recurrent CNVs that are very strong risk factors for the development of these disorders. ${ }^{5,6}$ It has now been shown that some CNVs confer risk for multiple neurodevelopmental disorders. For example duplication at the 16p11.2 locus increases risk for ID and ASD and has been shown to be associated with a 14 -fold increased risk of psychosis. ${ }^{7}$ Thus rare recurrent $\mathrm{CNVs}$ have broad, and often comorbid, neurodevelopmental phenotypes operating across traditional diagnostic boundaries.

Historically genetic investigation has not been a routine part of the assessment of adults with ID presenting to psychiatric services. ${ }^{8}$ However, the emergence of CNVs as important risk factors for multiple disorders raises the possibility that individuals with comorbid neurodevelopmental conditions should be prioritised where CMA testing is offered. We investigated the presence of undiagnosed likely pathogenic CNVs in adults with idiopathic ID presenting to community and in-patient psychiatric services in England. We discuss the frequency and type of CNVs identified; the associated phenotype and the implications of our findings.

\section{METHODS}

Study design and participant recruitment

Recruitment was undertaken via the Mental Health Research Network (MHRN) at 32 National Health Service (NHS) trusts and 1 non-NHS provider across England between August 2012 and March 2014. Consultant Psychiatrists in Intellectual Disabilities acted as local investigators at each site. Local investigators identified eligible participants from their caseloads based upon the study inclusion criteria, namely that the participants should be aged 18 years or older with idiopathic ID, one or more psychiatric diagnoses and/or significant challenging behaviours. Typically in the UK services use a high threshold for eligibility of ID services (IQ $<70$ as well as significant impairment of functioning which has been present from childhood). Idiopathic ID was defined as no clear genetic or environmental cause of ID detailed on the participant's medical records. Capacity to consent to the research project was assessed in accordance with the Mental Capacity Act 2005. Easy read information sheets and consent forms were utilised with individuals who had capacity to consent. In the absence of capacity consultees were identified to give advice as to the person's likely wishes regarding participation.

Clinical data including medical and psychiatric history (International Statistical Classification of Diseases and Related Health Problems (ICD-10) diagnoses), was collected from an informant and/or medical records. General observations for dysmorphic features were made and measurements of height and head circumference were collected by the clinician or researcher. Photographs were taken (where consent was given) for corroboration by the study team. Detailed psychiatric and behavioural phenotyping was undertaken using the Mini Psychiatric Assessment Schedule for Adults with Developmental Disabilities (Mini PAS-ADD) and Behaviour Problems Inventory - Short Form (BPI-S). The Mini PAS-ADD assesses psychiatric symptoms in seven diagnostic areas and provides threshold scores for symptoms that are likely to warrant a diagnosis in conjunction with a clinical assessment. ${ }^{9}$ The BPI-S provides frequency scores of behaviours on three domains, self-injurious behaviour, aggressive/destructive behaviour and stereotyped behaviour. ${ }^{10}$ Symptoms identified from Mini PAS-ADD or BPI-S screening are referred to as subclinical symptoms in the manuscript.

\section{Genetic analysis and feedback}

Participants provided either a blood (24\%) or saliva sample (76\%) for DNA extraction. aCGH analysis was undertaken at the North East Thames Regional Genetics Service Laboratory on the Nimblegen 135K platform. Arrays were processed and CNVs were reported using clinical diagnostic laboratory protocols, in keeping with the Association for Clinical Genetic Science (ACGS) best practice guidelines. ${ }^{11} \mathrm{CNVs}$ referred to in the manuscript as likely pathogenic include pathogenic causative findings and pathogenic susceptibility loci, both of which are thought to affect gene function in view of the associated phenotype. ${ }^{12}$ All likely pathogenic CNVs detailed in this manuscript were validated by qPCR, FISH or QF PCR and have been added to the Decipher genome browser. ${ }^{13}$ The other classes of CNV reported were variants of uncertain clinical significance (VOUS) and likely benign CNVs. Classification as VOUS includes: intronic CNVs, CNVs where no genes are present, CNVs where no entry was present on the Database of Genomic Variants (at the time of analysis), in addition to those where insufficient information is available to classify further. Further analysis has not been undertaken and these CNVs may include technical artefacts. Chromosomal abnormalities were also reported. Further analysis of relevant literature, and patient and control data sets was conducted by the research team and collaborators.

Likely pathogenic CNVs were fed back in writing to the participants' treating psychiatrist. The cytogenetic report detailing relevant CNVs and associated publications were provided alongside chromosomal disorder guides from the support group Unique where available. ${ }^{14}$ There is a paucity of appropriate and accessible information for adults with ID receiving diagnoses. The study team developed easy to read materials to aid feedback for some of the clinically relevant CNVs. Psychiatrists also had the opportunity to speak with a member of the research team regarding the result prior to feeding this back to their patients and family members and/or carers. Referral to the regional clinical genetics service was recommended for all likely pathogenic results. There were no adverse outcomes reported from feedback of the genetic test results.

\section{Statistical analysis}

Statistical analyses were undertaken using IBM SPSS Statistics for Windows, Version 22.0 (IBM Corp, Armonk, NY, USA). Univariate binary logistic regression was performed using Mini PAS-ADD thresholds and history of involuntary in-patient admission, including forensic in-patient section, as predictor variables. The binary outcome variable was presence or absence of a likely pathogenic CNV.

\section{RESULTS}

A total of 202 adults with idiopathic ID and comorbid psychiatric disorders/challenging behaviour were recruited to the study (63\% male; mean age 37 years, range 18-78 years; $74 \%$ White British). The yield of likely pathogenic CNVs, including chromosomal abnormalities, was $11 \%(22 / 202)$. A further $62 \%$ of participants had a least one CNV classed as a VOUS (126/202) and 27\% (54/202) had likely benign CNVs only. Details of the VOUS CNVs can be found in the Supplementary Information. An overview of likely pathogenic CNVs is presented in Figure 1, with detailed genetic and phenotypic data presented in Table 1 and Supplementary Table 1. A comparison of psychiatric diagnoses, subclinical symptoms and section history for likely pathogenic versus likely benign (including VOUS) CNVs is provided in Table 2. There were 21 participants on a forensic inpatient section at the time of recruitment and no other participants had a forensic section history. In all, 6/21 forensic in-patients carried likely pathogenic CNVs compared with $16 / 181$ in participants not on a forensic in-patient section. Thus the proportion of likely pathogenic $\mathrm{CNV}$ carriers in forensic in-patients was higher with an $\mathrm{OR}$ of 4.1 (95\% CI 1.40-12.04, $P=0.01$ ).

\section{Neurodevelopmental CNVs}

Most of the likely pathogenic CNVs were observed in regions of the genome prone to recurrent CNV. Five of these CNVs were identified at the 16p11.2 locus (4 duplications, 1 deletion) and one duplication at 16p13.11. The $16 \mathrm{p} 11.2$ region is associated with increased risk for ASD, schizophrenia and major depressive disorder, in keeping with the phenotypes observed in this cohort. ${ }^{15}$ A further five CNVs were identified in the 15q11.2-13.3 region (15q11.2 deletion, Angelman syndrome type 2, 15q12-13.1 deletion, 15q11.2-13.1 duplication and 15 q13.3 deletion) with variable psychiatric phenotypes. The CNVs at 


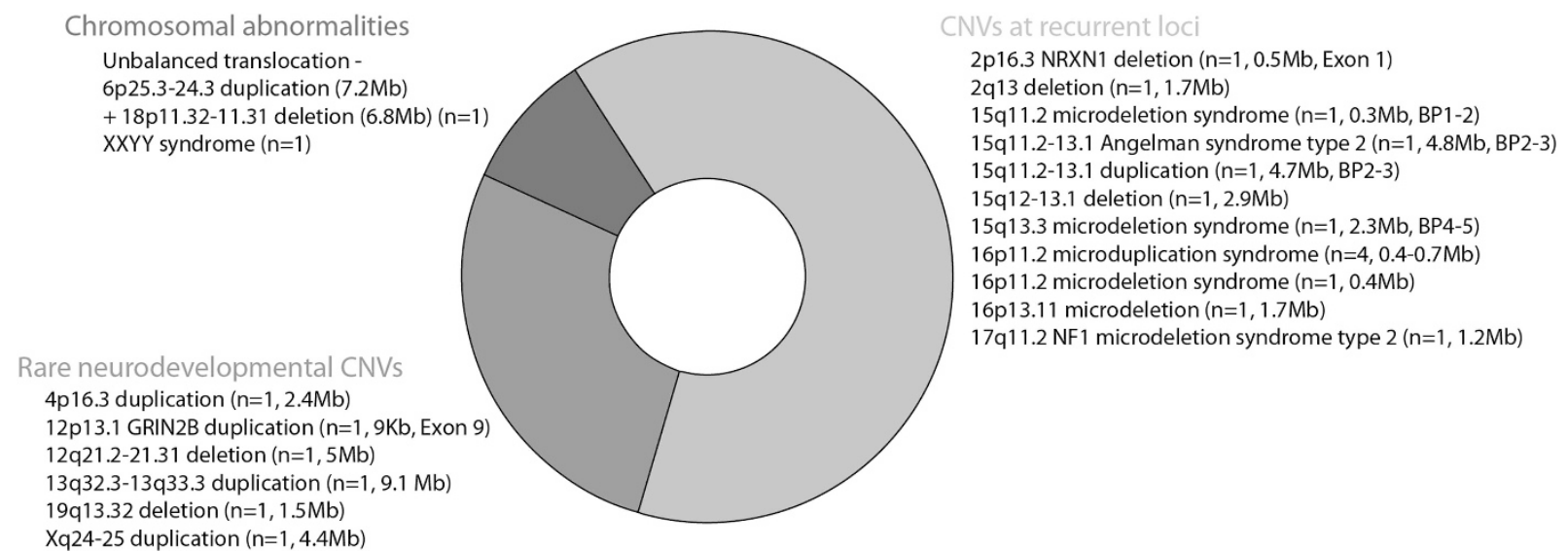

Figure 1 Overview of likely pathogenic CNVs identified in a sample of 202 adults with idiopathic intellectual disabilities and comorbid psychiatric disorders. Number of participants, approximate CNV size in megabases or kilobases (Mb or Kb), breakpoints (BP) and exon number are displayed in parenthesis as appropriate.

15q11.2, 15q13.3, 16p11.2 and 16p13.11 affect neurosusceptibility loci. These likely pathogenic CNVs have incomplete penetrance in that they occur at higher frequencies in disease cohorts, however, are not always associated with a disease phenotype and can sometimes be observed in healthy controls.

Another region prone to recurrent $\mathrm{CNV}$ is the 17q11.2 locus, which encompasses the neurofibromatosis type 1 (NF1) tumour suppressor gene. We identified a participant with a NF1 microdeletion presenting with a clinical diagnosis of ASD and challenging behaviours. This supports previous evidence of ASD being associated with variants in the NF1 gene. ${ }^{16}$ We also identified a deletion at $2 \mathrm{q} 13$ in a patient with a clinical diagnosis of ASD. Of the 29 patients described with this $\mathrm{CNV} 4$ are reported to have ASD. ${ }^{17} \mathrm{CNV}$ s in this region have also been shown to be enriched in schizophrenia cohorts, ${ }^{18}$ the participant presented with subclinical features of psychosis in addition to anxiety and behavioural problems. Two CNVs were identified in genes known to be important in neurodevelopment, namely GRIN2B and NRXN1. The GRIN2B gene is located at 12p13.1 and encodes the NR2 subunit of a $N$-methyl- $D$-aspartate glutamate receptor heteromer, which mediates excitatory neurotransmission and is thought to have an important role in memory and learning. Variants in the GRIN2B gene have previously been associated with behavioural problems. ${ }^{19} \mathrm{We}$ identified a duplication affecting exon 9 of the GRIN2B gene in a participant displaying self-injurious and aggressive behaviours. The NRXN1 gene is located at 2p16.3, it encodes a cell-surface receptor which is important for neurotransmission. Exonic NRXN1 deletions have been associated with increased risk for schizophrenia and ASD. ${ }^{20}$ Our participant has a deletion of exon 1 of the NRXN1 gene, a clinical diagnosis of personality disorder and subclinical symptoms of psychosis and stereotyped behaviour.

Five of the likely pathogenic CNVs identified have little or no prior association with psychiatric phenotypes in existing literature and likely represent rare emerging neurodevelopmental CNVs. First, we identified a duplication of $4 \mathrm{p} 16.3$, a region where deletions give rise to the better characterised Wolf-Hirschhorn syndrome. The CNV identified in our sample partially overlaps with the CNV reported in a case study of a patient with attention-deficit hyperactivity disorder (ADHD). ${ }^{21}$ Our participant had a clinical diagnosis of ASD and was a forensic in-patient. A duplication at Xq24-25 was observed in a participant with aggressive and stereotyped behaviours.
Abnormal behaviours, primarily hyperactivity, have previously been associated with CNVs in this region. The STAG2 gene, which encodes a component of the cohesion complex and is essential for chromosome segregation in dividing cells, has been identified as the likely causative gene. ${ }^{22}$

We identified a participant with a deletion at 12q21.2-21.31 comprising 17 genes. This region contains the Synaptotagmin-1 (SYT1) gene, which encodes a calcium-binding synaptic vesicle membrane protein involved in triggering neurotransmitter release at the synapse. A variant in SYT1, with a dominant negative function, has recently been associated with profound cognitive impairment. ${ }^{23}$ Whilst we observe a copy-number loss and different phenotype, a low haploinsufficiency score is suggestive of adverse functional consequences. $^{24}$ The participant has a clinical diagnosis of paranoid schizophrenia, a history of alcohol abuse and was a forensic in-patient. Another participant has a duplication at 13q32.3-13q33.3 comprising 33 genes. This region contains the D-amino acid oxidase activator $(D A O A)$ gene, which indirectly affects glutamatergic transmission and dopamine turnover. We and others have reported DAOA to be associated with both schizophrenia and bipolar affective disorder. ${ }^{25}$ The participant has a clinical diagnosis of ASD and ADHD, and is a forensic in-patient. Finally, we identified a deletion at $19 \mathrm{q} 13.32$ comprising 56 genes. This deletion partially overlaps with a case reported previously but does not include any of the proposed candidate genes. ${ }^{26}$ The participant has a clinical diagnosis of depression and bipolar affective disorder.

\section{DISCUSSION}

Approximately $11 \%$ new diagnoses could be made by testing adults with ID accessing psychiatry services in the UK. Recently there have been calls for increased clinical use of CMA in patients with schizophrenia. The yield of likely pathogenic CNVs in adults with schizophrenia is reported to be in the range of $2.5-5 \% .{ }^{27,28}$ Our higher diagnostic yield argues that clinicians should also consider adopting routine CMA in ID psychiatry. Interestingly the most frequently observed $\mathrm{CNV}$ in this study was the $16 \mathrm{p} 11.2$ duplication (4 individuals, $2 \%$ ). This CNV has been widely reported in other studies, with a frequency of $\sim 0.2 \%$ in $\mathrm{DD} / \mathrm{ID},{ }^{29} \sim 1 \%$ in $\mathrm{ASD}^{5}$ and $\sim 0.3 \%$ in schizophrenia. ${ }^{28}$ Accepting the small sample size this may suggest a particular enrichment of this recurrent $\mathrm{CNV}$ in the adult 


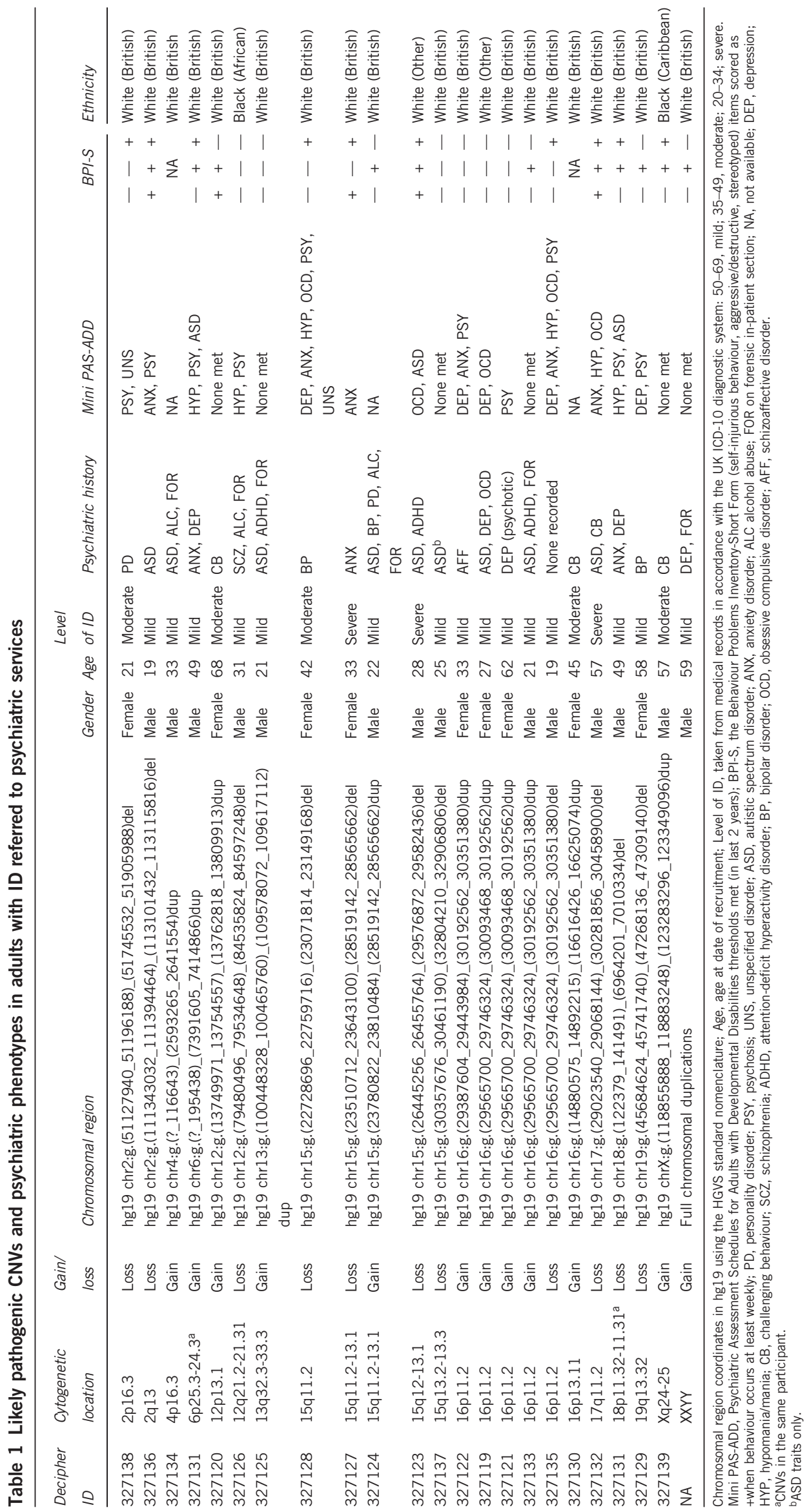


Table 2 Psychiatric phenotype (ICD-10 diagnoses, Mini PAS-ADD thresholds and section history) for likely pathogenic and benign CNVs

\begin{tabular}{|c|c|c|c|}
\hline & $\begin{array}{l}\text { Total in sample (\%) } \\
\qquad \mathrm{n}=202\end{array}$ & $\begin{array}{l}\text { Likely pathogenic CNV group (\%) } \\
\qquad \mathrm{n}=22\end{array}$ & $\begin{array}{l}\text { Likely benign CNV group (\%) } \\
\qquad \mathrm{n}=180\end{array}$ \\
\hline \multicolumn{4}{|l|}{ ICD-10 diagnosis } \\
\hline Bipolar disorder & $23(11 \%)$ & $3(14 \%)$ & $20(11 \%)$ \\
\hline Depressive episode & $62(31 \%)$ & $4(18 \%)$ & $58(32 \%)$ \\
\hline Other anxiety disorders & $45(23 \%)$ & $2(9 \%)$ & $43(24 \%)$ \\
\hline \multicolumn{4}{|l|}{ Mini PAS-ADD thresholds } \\
\hline Psychosis & $72(36 \%)$ & $9(41 \%)$ & $63(35 \%)$ \\
\hline Hypomania/mania & $33(16 \%)$ & $5(23 \%)$ & $28(16 \%)$ \\
\hline Depressive disorder & $76(38 \%)$ & $5(23 \%)$ & $71(39 \%)$ \\
\hline Anxiety disorder & $80(40 \%)$ & $6(27 \%)$ & $74(41 \%)$ \\
\hline Previous history of involuntary admission & $45(22 \%)$ & $7(32 \%)$ & $38(21 \%)$ \\
\hline Forensic section & $21(10 \%)$ & $6(27 \%)$ & $15(8 \%)$ \\
\hline
\end{tabular}

ICD-10 diagnoses - the psychosis group was amalgamated to comprise: F20 schizophrenia, F25 schizoaffective disorder and F29 unspecified nonorganic psychosis. Other ICD-10 diagnoses reported independently are: F31 bipolar disorder, F32 depressive episode, F41 other anxiety disorders, F90 hyperkinetic disorder, F84 pervasive developmental disorder. Mini PAS-ADD thresholds - scores were calculated using standard guidelines, Mental Health Act (MHA) section - previous history of involuntary admission included previous and current MHA sections and forensic sections, forensic section - all individuals were on a forensic section at the time of recruitment no history of being on a forensic section was identified in any of the other participants. Note: several individuals had comorbid diagnoses and are included in more than one category.

population of ID and comorbid psychiatric disorder. The yield of VOUS CNVs in the study also appears to be high at $62 \%$. This is of considerable interest for future research, although it is difficult to determine whether VOUS are enriched in this cohort due to lack of comparable data sets.

A broad range of psychiatric diagnoses/symptoms were observed across the cohort. The pattern of comorbidities, either defined by ICD-10 diagnoses or Mini PAS-ADD thresholds, was complex. Inclusion of Mini PAS-ADD thresholds indicated a burden of psychopathology not captured by ICD-10 diagnoses. It is of interest that $41 \%$ of participants with likely pathogenic CNVs met the Mini PAS-ADD threshold for psychosis, whereas based upon ICD-10 criteria this was only $14 \%$, see Table 2 . Overall psychiatric features did not differentiate the likely pathogenic and likely benign CNV groups. However there was an excess of participants on a forensic section in the likely pathogenic CNV group in comparison with the benign (including VOUS) CNV group. No link can be made between specific CNVs and offending behaviour, as causality cannot be inferred. The population of people with ID and convictions is particularly marginalised and it may be that increased barriers to genetic testing have resulted in a higher prevalence of undiagnosed CNVs. This finding warrants further investigation in much larger samples. Assessment of adults with ID and formulation of the psychiatric presentation can be challenging. The majority of likely pathogenic CNVs were found at recurrent $\mathrm{CNV}$ loci where, at least some, information on the associated phenotype is available. Such information may aid understanding of the patient's clinical presentation for both clinicians and family members. Furthermore knowledge of associated phenotypes may guide psychiatric evaluation. For example the identification of a 16p11.2 duplication would be an indicator to screen for the presence of ASD, psychosis or affective disorders. ${ }^{15}$
Importantly some of the CNV syndromes are also associated with non-psychiatric comorbidities that require specific medical management or monitoring. For example we identified an individual with undiagnosed Angelman's syndrome type 2 and an individual with an NF1 deletion. Comprehensive medical guidelines are available for Angelman's syndrome and NF1 deletions. ${ }^{30}$ The characterisation of very rare CNVs is an ongoing process and clinical guidelines are being developed as new syndromes continue to emerge. However, some diagnoses will have minimal impact on patient management.

There is evidence for the benefit to mothers in receiving a diagnosis for a child with DD/ID. ${ }^{31}$ However, little is known about the impact of disclosure of a genetic diagnosis to adults with ID or their families/carers. Challenges are faced with ascertaining capacity to consent to genetic testing, feedback of diagnoses and possible incidental findings. We received no negative feedback from psychiatrists in this study about the diagnostic process. All participants with likely pathogenic $\mathrm{CNV}$ s were sent information about a rare chromosomal support group called Unique; this group is only accessible to individuals with a diagnosis. ${ }^{14}$ Genetic diagnosis of the affected individual provides the opportunity for cascade testing of at risk relatives and the provision of recurrence risk information. The inheritance of a likely pathogenic CNV in a child with associated difficulties may be important in supporting an application for a statement of special educational needs.

This study has several specific limitations. The sample size was modest. The recruitment strategy focused on individuals with a more severe psychiatric phenotype, that is, those presenting to psychiatric services. We may have under sampled those with the most severe phenotypes because of difficulties recruiting this population group to research studies. However, our sample is likely representative of individuals accessing specialist services 
for ID and comorbid mental illness. Estimates of the penetrance of particular phenotypes would require epidemiological based studies. Another major limitation is the lack of inheritance status, which is often used to inform interpretation of rare variants. Technological limitations of the aCGH platform include inability to detect balanced translocations, single-gene disorders and lowlevel mosaicism. As the array platform has not been utilised in research studies of control populations comparisons with other studies is prone to technical confounds. Exome and whole-genome sequencing, which is gradually coming into clinical practice, is likely to increase the yield of genetic diagnoses for those with ID and comorbid psychiatric disorders.

\section{CONCLUSIONS}

We have demonstrated that CNV screening using clinically available CMA offers over one in ten new aetiological diagnoses for adults with idiopathic ID presenting to psychiatric services in the UK. It may be appropriate for ID psychiatrists to offer CMA more routinely in assessment of people with ID and comorbid mental health problems, particularly in forensic settings. Liaison between clinicians and genetic services will be important for the interpretation of new genomic investigations in clinical practice. ${ }^{32}$

Clinical and research data on emerging CNV syndromes are strongly biased towards paediatric populations. However, the full extent of the phenotype associated with a particular $\mathrm{CNV}$ may only be realised in adulthood as psychiatric disorders emerge. We found the $16 \mathrm{p} 11.2$ duplication to be particularly frequent in this understudied adult population. We also add psychiatric phenotypic information to very rarely observed and novel likely pathogenic CNVs. CMA testing in adults could potentially inform the clinical management of children with new emerging likely pathogenic CNVs.

\section{CONFLICT OF INTEREST}

The authors declare no conflict of interest.

\section{ACKNOWLEDGEMENTS}

Unique, Participating research sites from the NIHR MHRN (Avon \& Wiltshire Mental Health Partnership NHS Trust, Barnet, Enfield and Haringey Mental Health NHS Trust, Berkshire Healthcare NHS Foundation Trust, Birmingham and Solihull Mental health NHS Foundation Trust, Black Country Partnership NHS Foundation Trust, Bradford District Care Trust, Cambridgeshire and Peterborough NHS Foundation Trust, Camden \& Islington Mental Health and Social Care Trust London, Central \& North West London NHS Foundation Trust, Cheshire and Wirral Partnership NHS Foundation Trust, Cornwall Partnership NHS Trust, Cumbria Partnership NHS Foundation Trust, Derbyshire Healthcare NHS Foundation Trust, East London NHS Foundation Trust, Humber Mental Health Teaching NHS Trust, Kent and Medway NHS and Social Care Partnership Trust, Leicestershire Partnership NHS Trust, Manchester Mental Health \& Social Care NHS Trust, North Essex Mental Health Partnership NHS Trust, North Staffordshire Combined Healthcare NHS Trust, Northamptonshire Healthcare NHS Trust, Northumberland, Tyne and Wear NHS Trust, Nottinghamshire Healthcare NHS Trust, Somerset Partnership NHS Foundation Trust, South Essex Partnership University NHS Foundation Trust, South West London and St Georges Mental Health NHS Trust, South West Yorkshire Partnership NHS Foundation Trust, South West Yorkshire Partnership NHS Foundation Trust, Southern Health NHS Foundation Trust, St Andrews Healthcare, St James Hospital-Solent NHS, Surrey and Borders Partnership NHS Foundation Trust, Tees, Esk and Wear Valleys NHS Foundation Trust). North East Thames Regional Genetics Service Laboratory staff, Dr Nick Lench and Dr Jonathan Waters. Role of funding source: Baily Thomas
Charitable Fund (funding for participant recruitment). The Medical Research Council funded 4-year PhD in Mental Health (Kate Wolfe). Ethics: North Wales Research Ethics Committee West, reference 11/WA/0370

\section{AUTHOR CONTRIBUTIONS}

Literature search, study design, data collection, data analysis, data interpretation, writing of the manuscript: KW, AS, NB and AM. Participant recruitment, data collection and critical review of the manuscript: $\mathrm{PC}, \mathrm{ME}, \mathrm{AH}$, JM, RM, DP, NP, SR, RS, SS and ST. Data analysis and critical review of the manuscript: DM, JC, JT, CP, CO and FF.

1 Palmer E, Speirs H, Taylor PJ et al: Changing interpretation of chromosomal microarray over time in a community cohort with intellectual disability. Am J Med Genet A 2014; 164: 377-385.

2 Cooper S-A, Smiley E, Morrison J et al: Psychosis and adults with intellectual disabilities. Prevalence, incidence, and related factors. Soc Psychiatry Psychiatr Epidemiol 2007; 42: 530-536.

3 Miller DT, Adam MP, Aradhya S et al: Consensus statement: chromosomal microarray is a first-tier clinical diagnostic test for individuals with developmental disabilities or congenital anomalies. Am J Hum Genet 2010; 86: 749-764.

4 lyer J, Girirajan S: Gene discovery and functional assessment of rare copy-number variants in neurodevelopmental disorders. Brief Funct Genomics 2015; 14 315-328.

5 Weiss LA, Shen YP, Korn JM et al: Association between microdeletion and microduplication at 16p11.2 and autism. N Engl J Med 2008; 358: 667-675.

6 Kirov G, Gumus D, Chen W et al: Comparative genome hybridization suggests a role for NRXN1 and APBA2 in schizophrenia. Hum Mol Genet 2008; 17 458-465.

7 Giaroli G, Bass N, Strydom A et al: Does rare matter? Copy number variants at $16 \mathrm{p} 11.2$ and the risk of psychosis: a systematic review of literature and meta-analysis. Schizophr Res 2014; 159: 340-346.

8 De Villiers J, Porteous M: Genetic testing of adults with intellectual disability. Psychiatrist 2012; 36: 409-413.

9 Moss S, Prosser $\mathrm{H}$, Costello $\mathrm{H}$ et al: The mini PAS-ADD: An assessment schedule for the detection of mental health needs in adults with learning disability (mental retardation), Hester Adrian Research Centre. University of Manchester: Manchester, UK, 1997.

10 Rojahn J, Rowe EW, Sharber AC et al: The Behavior Problems Inventory-Short Form for individuals with intellectual disabilities: part II: reliability and validity. J Intellect Disabil Res 2012; 56: 546-565.

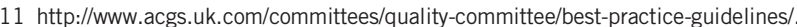

12 Srebniak MI, Diderich KEM, Govaerts LCP et al: Types of array findings detectable in cytogenetic diagnosis: a proposal for a generic classification. Eur J Hum Genet 2014; 22: $856-858$

$13 \mathrm{https} / / / \mathrm{dec} i p h e r . s a n g e r . a c . u k / b r o w s e r$

$14 \mathrm{http}: / / \mathrm{www}$.rarechromo.co.uk/html/home.asp.

15 Degenhardt F, Priebe L, Herms S et al: Association between copy number variants in 16 p11.2 and major depressive disorder in a German case-control sample. Am J Med Genet Part B Neuropsychiatr Genet 2012; 159 B: 263-273.

16 Garg S, Plasschaert E, Descheemaeker M-J et al: Autism spectrum disorder profile in neurofibromatosis type I. J Autism Dev Disord 2015; 45: 1649-1657.

17 Hladilkova $\mathrm{E}$, Barøy $\mathrm{T}$, Fannemel $\mathrm{M}$ et al: A recurrent deletion on chromosome $2 \mathrm{q} 13$ is associated with developmental delay and mild facial dysmorphisms. Mol Cytogenet 2015; 8: 57.

18 Costain G, Lionel AC, Merico D et al: Pathogenic rare copy number variants in community-based schizophrenia suggest a potential role for clinical microarrays. Hum Mol Genet 2013; 22: 4485-4501.

19 Freunscht I, Popp B, Blank R et al: Behavioral phenotype in five individuals with de novo mutations within the GRIN2B gene. Behav Brain Funct 2013; 9: 20.

20 Viñas-Jornet M, Esteba-Castillo S, Gabau E et al: A commoncognitive, psychiatric, and dysmorphic phenotype in carriers of NRXN1 deletion. Mol Genet Genomic Med 2014; 2: $512-521$.

21 Palumbo O, Palumbo P, Ferri $\mathrm{E}$ et al: Report of a patient and further clinical and molecular characterization of interstitial 4p16.3 microduplication. Mol Cytogenet 2015; 8: 1-5.

22 Leroy $C$, Jacquemont M-L, Doray B et al: Xq25 duplication: the crucial role of the STAG2 gene in this novel human cohesinopathy. Clin Genet 2015.

23 Baker K, Gordon SL, Grozeva D et al: Identification of a human synaptotagmin-1 mutation that perturbs synaptic vesicle cycling. J Clin Invest 2015; 125: 1-9.

24 Huang N, Lee I, Marcotte EM et al: Characterising and predicting haploinsufficiency in the human genome. PLoS Genet 2010; 6: 1-11.

25 Bass NJ, Datta SR, McQuillin A et al: Evidence for the association of the DAOA (G72) gene with schizophrenia and bipolar disorder but not for the association of the DAO gene with schizophrenia. Behav Brain Funct 2009; 5: 28.

26 Castillo A, Kramer N, Schwartz CE et al: 19q13.32 microdeletion syndrome: three new cases. Eur J Med Genet Elsevier Masson SAS 2014; 57: 654-658. 
27 Baker K, Costain G, Fung WLA et al: Chromosomal microarray analysis-a routine clinical genetic test for patients with schizophrenia. Lancet Psychiatry 2014; 1: 329-331.

28 Rees E, Walters JTR, Georgieva L et al: Analysis of copy number variations at 15 schizophrenia-associated loci. Br J Psychiatry 2014; 204: 108-114.

29 Cooper GM, Coe BP, Girirajan S et al: A copy number variation morbidity map of developmental delay. Nat Genet 2011; 43: 838-846.

$30 \mathrm{http}: / /$ www.orpha.net/consor/cgi-bin/Disease.php.

31 Lingen M, Albers L, Borchers $M$ et al: Obtaining a genetic diagnosis in a child with disability: impact on parental quality of life. Clin Genet 2015; 89: 258-266.

32 Kharbanda M, Tolmie J, Joss S: How to use... microarray comparative genomic hybridisation to investigate developmental disorders. Arch Dis Child Educ Pract Ed 2015; 100: 24-29.
This work is licensed under a Creative Commons Attribution 4.0 International License. The images or other third party material in this article are included in the article's Creative Commons license, unless indicated otherwise in the credit line; if the material is not included under the Creative Commons license, users will need to obtain permission from the license holder to reproduce the material. To view a copy of this license, visit http:// creativecommons.org/licenses/by/4.0/

(C) The Author(s) 2017

Supplementary Information accompanies this paper on European Journal of Human Genetics website (http://www.nature.com/ejhg) 